\title{
Number of pregnant adolescent women who come for first antenatal care within 16 weeks of gestational age and the total numbers of antenatal care visits during pregnancy compared to the other group
}

\author{
Article by Lindiwe G. Mhlanga \\ Master of Public Health, Texila American University, Swaziland \\ Email: lgmhlanga@ymail.com
}

\section{Introduction}

Labour and delivery is the end of pregnancy and beginning of new life. Antenatal care plays an important role to achieve a successful labour and delivery process. Regular antenatal classes help in the physical and mental preparation of women and help them relax during those last months. Antenatal care ensures maternal fetal health wellbeing and also prepares women physically fit for labour, delivery and the postpartum period. ${ }^{1}$

Nighty four percent of women access antenatal services in Swaziland at least once and seventy one percent of pregnant women deliver in health facility. ii A majority of the country's population is young. According to Swaziland's latest census report (2007), 52\% of the 1.1 million people are under the age of 20 and those aged $10-24$ years constitute $37 \%$ of the population (39\% male and $36 \%$ female) $)^{2}$. In addition, adolescents comprise of $37 \%$ of the country's population among which $25 \%$ of 33,000 deliveries areadolescents. $27 \%$ of antenatal care (ANC) clients who live with HIV areadolescents ${ }^{3}$.

Maternal mortality is still high in Swaziland where we lose a significant number pregnant women, it accounts to 320/100 000 live births. ${ }^{4}$ Female adolescents account for a significant proportion of maternal deaths, which are largely due to preventable causes like malnutrition, infections and hemorrhage coupled with inadequate health care negligence and supportive services, particularly in the rural areas. Unsafe abortions contribute significantly to maternal morbidity and mortality among adolescents.

The Ministry of health through the Sexual and Reproductive Health Unit has adopted

\section{Purpose}

The purpose of the study was review if there is any improvement in the number of pregnant women who attend antenatal care as indicated in the national guidelines.

It also wanted to understand if the number of adolescent mothers who come within 16 weeks of gestation is improving. As these can significantly improve maternal mortality rate because most of the contributing factors to maternal mortality are preventable.

Finally the study wanted to find out if the pregnant women are adhering to the four focused visits adopted by the Ministry of health during antenatal care which also can improve maternal health.

\section{Methods}

Data abstraction was conducted in one facility (Silele Red Cross clinic, Shiselweni Region) for all women who came for antenatal care from January to March 2015. When the data was abstracted in October 2015 all the pregnant mothers were expected to have delivered. The aim was to compare if there is any significance to the number of antenatal visit for pregnant women more especially to the adolescent women as they are more at risk for maternal deaths.

\section{Results}

From January to March 2015 only 46 women came for first antenatal care visit, 19/46 were adolescent (forty one percent). Those who came at weeks of gestation for earlier were only 15 (thirty two percent of all pregnant women), for adolescents 6/15 (forty percent). Overall 
South American Journal of Public Health

Special Edition May 2016

women who were able to reach the four focused visits were 13/46, which accounts for twenty eight percent.

Below is the table highlighting the number who attended antenatal care according to age category.

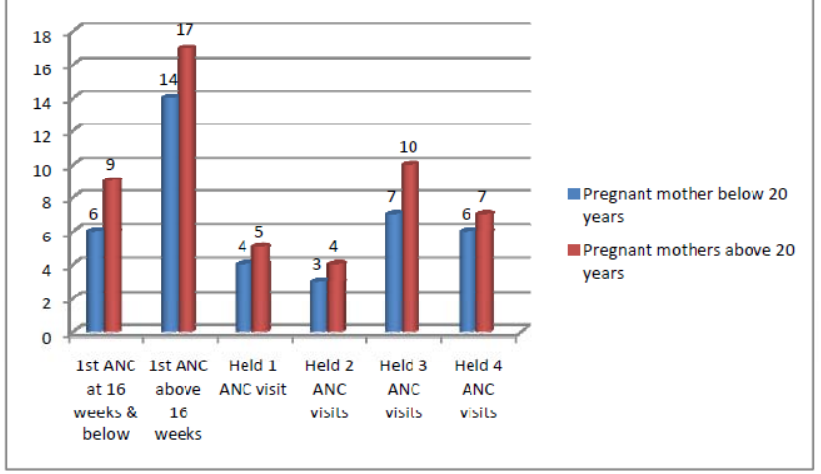

\section{Discussions/ Conclusions}

Though nighty four percent of women access antenatal services in Swaziland at least once and seventy one percent of pregnant women deliver in health facility, ii this indicates that at least once during pregnancy women for the health services, but this is not enough as other conditions need to be detected early to good prognoses. About $41 \%$ of pregnant women in Swaziland are infected with HIV, and they give birth to over 17000 HIV-exposed infants each year ${ }^{5}$. If a majority of pregnant mother will not come within 16 weeks of gestational age other diseases like HIV may be transmitted to the unborn child.

Still there is a gap for pregnant mothers to access services as recommended by the Ministry of health thus improve maternal mortality.

There is a need to access the teenage mothers to give more information on the importance of antenatal care visits, for their health as well as their unborn babies.

\section{References}

[1.] Central Statistical Office. Swaziland Population and Housing Census, Mbabane, Swaziland. 2007. http://drsimran.expertscolumn.com/article/why-antenatal-care-important-during-pregnancy. Abstracted October 15, 2015 at 08.10hrs.

[3.] http://www.indexmundi.com/swaziland/maternal_mortality_rate.html. Abstracted October 15, 2015 at 08.18 Hrs.

[4.] Swaziland. Ministry of Health. National Sexual Reproductive Health and Rights Strategic Plan 2014 - 2018. Mbabane. 2015, p10-11.

[5.] Swaziland. Ministry of Health. 11th National HIV sero-surveillance among women attending antenatal care services. Mbabane: Printpak. 2010 